\title{
Organizational transformation to integrated employment and community life engagement
}

\author{
Jennifer Sullivan Sulewski ${ }^{\mathrm{a}, *}$, Jaimie Ciulla Timmons ${ }^{\mathrm{a}}$, Oliver Lyons ${ }^{\mathrm{a}}$, Jonathan Lucas ${ }^{\mathrm{b}}$, \\ Tim $\operatorname{Vogt}^{\mathrm{c}}$ and Katie Bachmeyer ${ }^{\mathrm{c}}$ \\ ${ }^{a}$ Institute for Community Inclusion, University of Massachusetts Boston, MA, USA \\ ${ }^{\mathrm{b}}$ The Arc of the United States, Washington, DC, USA \\ ${ }^{\mathrm{c}}$ Starfire Council, Cincinnati, OH, USA
}

Revised/Accepted November 2016

\begin{abstract}
.
BACKGROUND: States have moved toward defunding sheltered workshops and supporting the transformation towards integrated competitive employment and inclusive day services. As a result, many local service providers have been challenged to consider how they will transform their services in order to provide fully inclusive work and community life engagement opportunities.

OBJECTIVE: Findings from a Delphi panel of experts in the field of organizational transformation offer ten elements necessary for successful change away from segregated work options. Findings from expert interviews on community life engagement indicate four guideposts for the provision of high-quality non-work day supports.

CONCLUSION: Combining findings across these two projects, in addition to a reflection from a provider that has undergone transformation, this manuscript offers guidance for providers of day and employment services in their efforts to support competitive integrated employment and meaningful community life engagement for individuals with intellectual and developmental disabilities.
\end{abstract}

Keywords: Integrated employment, community life engagement, transformation, IDD

\section{Introduction}

Within the last ten years, the federal government has increasingly promoted the inclusion of people with disabilities in their communities, and in all facets of life. A key turning point was the 1999 Olmstead v. L.C. decision, in which it was

\footnotetext{
*Address for correspondence: Jennifer Sullivan Sulewski, Institute for Community Inclusion, University of Massachusetts Boston, 100 Morrissey Blvd., Boston, MA 02125, USA. Tel.: +1 617287 4356; Fax: +1 617287 4352; E-mail: jennifer. sulewski@umb.edu.
}

held that segregated services for people with disabilities violated the Americans with Disabilities Act. In 2009 through direct orders from President Obama, a reinforcement of this decision was carried out (The White House, 2011). States are now required to eliminate unnecessary segregation of persons with disabilities and to ensure that they receive services in the most integrated setting appropriate to their needs. Beginning in 2012, several states including Oregon and Rhode Island settled class-action lawsuits and Olmstead investigations finding unnecessary segregation of individuals with intellectual and 
developmental disabilities (IDD) in day programs and sheltered workshops. As a result, the Department of Justice has released related guidance around the provision of day and employment supports in integrated settings (United States v. State of Rhode Island, 2014; U.S. Department of Justice, 2011) that has illustrated the need to define and provide high-quality community-based employment and day supports. During the same time period, the Centers for Medicare and Medicaid Services released new rules that defined, described, and aligned home and community-based setting requirements specifying maximum opportunities in the most integrated settings (Centers for Medicare and Medicaid Services, 2014).

As a result of this shift, states have moved toward defunding sheltered workshops and supporting the transformation towards integrated competitive employment and integrated day services for those individuals. Consequently, local service providers have been challenged to consider how they will transform their facility-based employment and day services in order to provide opportunities for competitive integrated employment and community life engagement supports.

Competitive integrated employment is defined within the Workforce Innovation and Opportunity Act as full-time or part-time work at minimum wage or higher, with wages and benefits similar to those without disabilities performing the same work, and fully integrated with co-workers without disabilities (Hoff, 2014). Community life engagement (CLE) refers to how people with IDD access and participate in their communities outside of employment as part of a meaningful day. CLE supports may be referred to as Community-Based Non-Work, wraparound supports, holistic supports, or community integration services. CLE activities may include volunteer work; postsecondary, adult, or continuing education; accessing community facilities such as a local library, gym, or recreation center; participation in retirement or senior activities; and anything else people with and without disabilities do in their off-work time.

As states and providers struggle with the challenges associated with transformation, research continues to suggest variation of services and employment philosophies within the provider community. Inge et al. (2009) found that almost $89 \%$ of respondents to a national survey of provider administrators believed that facility-based programs were essential for individuals with disabilities who are hav- ing difficulty getting or maintaining real work in the labor force, and less than half of these administrators had a formal plan to expand integrated employment. Providers perceived inadequate funding and community resources to provide individual employment. Within the organizations themselves, front-line staff experienced confusion about job development responsibilities, did not feel prepared to engage the mainstream business community, and had little training in providing appropriate supports to individuals with IDD in community settings. This manuscript considers such service provider challenges and offers guidelines to support organizational transformation efforts. The paper will share (1) research findings related to ten essential elements necessary for successful organizational transformation toward integrated employment, (2) research findings related to four guideposts for high-quality CLE supports; and (3) the experiences of a service provider that has successfully transformed to integrated day and employment supports.

\subsection{Research project on organizational transformation}

The goal of ICI's organizational transformation initiative is to develop a strategy for supporting service providers to rebalance resources to emphasize integrated employment. The project used a Delphi process (see Methods section) to identify, define, and refine essential characteristics present in successful transformation efforts (see Results section). The Delphi process is being followed by case study research of service providers that have successfully transformed services to understand on-the-ground implementation. Findings from this research will be used to develop a framework and toolkit to be used a blueprint to guide providers in the process.

\subsection{Research project on community life engagement}

The Community Life Engagement project has included a series of expert interviews (see Methods section), case studies of three exemplary providers of CLE supports, and the identification of several promising practices in CLE. The combination of these activities led to the development of four guideposts (see Results section) and a toolkit for states and service providers on how to design, conduct, regulate, and measure high-quality CLE. 


\subsection{Starfire: The transformation experience of one service provider}

In 1993, a group of volunteers and parents founded Starfire in the hope that people with developmental disabilities (DD) could have a more rich social life. They formed an outing program that offered social activities for people with DD to choose from, with trips led by a staff person and 1-2 volunteers and accommodating up to 10 people with DD at a time. Starfire also originally operated two facility-based day programs, where people with DD participated in "life skills" activities such as cooking, exercise, and day trips in the community. At its peak, Starfire served up to 600 people with DD and ran 100 outings per month, including the two full-time day programs.

In 2009, Starfire began a process of discovery through the teachings of Dr. Wolf Wolfensburger and Dr. John O'Brien that led to an important realization: these segregated programs were not leading to sustainable, lifelong relationships or a true sense of community belonging. Consequently, in 2010 Starfire formed a new Strategic Plan that focused on transforming the organization away from congregated services to fully integrated, one-on-one supports that focus on each person's gifts and distinct contributions. Today, Starfire's mission is building a sense of belonging and purpose through relationships and valued social roles in the community, one person at a time.

Starfire's model has these key aspects: (1) Personcentered Planning - to assist people with DD to plan their lives by focusing on their strengths; (2) Normalization (W. Wolfensberger) - people with DD must be seen as valued, respected citizens first in order to connect with the community; (3) AssetBased Community Development (J. McKnight) leveraging free resources and local assets as the primary building blocks of sustainable community; (4) "Five Valued Experiences" (J. O'Brien) - A service model that states all citizens have better lives when they have opportunities to share places, make choices, experience respect, grow in relationships, and make contributions.

On September 16, 2016, Starfire completed a 7year transformation from segregated programming to an organization that provides direct supports in the community. Starfire is now building up to serve 100 people with DD in this new way, with current program membership at 73 . Rather than aiming to reach hundreds of people and providing group supports, the organization believes it can serve each person in a deeper way through a smaller, more localized approach.

\section{Methods}

\subsection{Research on organizational transformation}

A Delphi process was used to identify, understand, and prioritize the most important elements of organizational change. The Delphi method is a "social research technique whose aim is to obtain the most reliable group consensus of a group of experts," (Linstone \& Turoff, 1975 p.10). It differs from other qualitative group-based research methods in four distinct ways: (1) the process ensures anonymity for all respondents; (2) the interview process is iterative, which provides opportunity for continuous and controlled feedback; (3) the method allows researchers to capture data that is statistically interpretable; and (4) the possibility of using email or online surveys as a means to communicate and gather information allows for participants to be geographically distributed (Lindqvist \& Nordänger, 2007). Because the Delphi method does not rely on creating a physical group setting for research, the process can involve more individuals than can effectively interact in a face-to-face group setting, which allows for an increased sample size and heterogeneity in the respondent group.

\subsubsection{Sample}

The Delphi panel members represented a range of stakeholder groups, including provider administrators and leadership, trainers and technical assistance providers, state IDD agency staff, direct support professionals, family members, individuals with IDD, and researchers. The respondents had knowledge and experience in either leading or participating in an organizational transformation process. Of the 36 Delphi panelists, 26 reported having taken part in an organizational change process, 20 had served in a leadership role in a transformation process, and 28 had participated in organizational transformation planning process.

\subsubsection{Data collection}

The data for the study was collected via two successive online surveys administered in July and December, 2015. The first survey was administered to seek feedback on six characteristics essential in organizational transformation identified by earlier research (Butterworth, Gandolfo, Revell, \& Inge, 
2007) and add any essential elements they felt were missing after reviewing the original six. The second survey asked the same respondents to rank existing and new characteristics based on level of importance to organizational transformation.

\subsection{Community life engagement}

\subsubsection{Sample}

Experts were chosen based on their level of expertise and diversity of perspectives. Two were self-advocates, 5 were family members of people with IDD, 4 were service provider executives, 2 were state agency executives, and 2 were researchers. Some participants represented more than one role (e.g. both a provider executive and a parent of an adult with IDD).

\subsubsection{Data collection}

Each expert participated in a 45- to 90-minute semi-structured telephone interview. Topics covered included the goals of CLE, evidence of effective implementation of CLE, barriers encountered and strategies used, and the role of CLE as a support to other outcomes, including employment. All interviews were recorded and transcribed, and the transcripts were then reviewed by the project team to identify common themes.

\subsection{Starfire's outcomes tracking}

Starfire collects data from each person supported at his/her orientation and every 6 months moving forward. Starfire's continuous learning model is based on research by The Council on Quality and Leadership (CQL), a national accreditation organization for disability services (The Council on Quality and Leadership, 2014), along with Dr. Angela Amado's "Friends" manual (Amado, 2013), both of which instruct and measure disability services on connecting people with disabilities to the community. The person-centered tools used to collect data at Starfire were developed by Dr. Amado and Dr. John O'Brien and adapted to Starfire's uses.

\section{Results}

\subsection{Organizational transformation}

According to Delphi panelists, in order to transform a facility-based program to one that supports individuals in competitive integrated employment, a service provider must have the following ten elements in place. The panelists ranked them in order of their importance to the field.

Clear and consistent goals: An explicit commitment to increasing competitive integrated employment is essential. Goals must be measurable, compelling, and easy to grasp, directly reflective of the core mission, modifiable, and specific to an established time frame. They must also reflect the needs of individuals.

An agency culture that values inclusion: Agencies need to establish a culture that values supporting individuals in the community rather than in facilities. The culture also must value positive thinking, learning, creativity, innovation, and continuous quality improvement.

An active, person-centered job placement process: At the same time that they are focused on changes at the organizational level, agencies must be proactive in moving forward at the individual level, finding jobs for one person at a time. This "just do it" approach creates momentum and enthusiasm as successful employment outcomes are achieved and celebrated.

A strong internal and external communications plan: Providers must communicate clear, authentic expectations for competitive integrated employment. Internally, this includes all levels of staff, individuals, and their families. Externally, successful organizations market themselves and their services throughout the community.

Reallocated and restructured resources: Transformation requires an active and ongoing investment in realigning all fiscal, material, and staff resources in order to put into place the supports and services needed for increasing competitive integrated employment.

An ongoing investment in staff professional development: Essential to the transformation is frequent and ongoing training, continuing education, conference participation, and mentorship opportunities to develop and maintain staff's core competencies and to implement best practices.

A focus on customer engagement: Providers must engage with their customer groups. First, they can partner with self-advocates, families, funders, and other community partners. Second, they can engage new and existing business partners to meet both individual and market needs.

Effective employment performance measurement, quality assurance, and program oversight: Establishing a clear framework for implementing and 
measuring administrative, management, and program strategies over defined periods of time will help agencies determine the impact of their efforts and their success in obtaining desired results.

A holistic approach: Providers must consider the whole person with wrap-around life supports as necessary, and use a career planning process that involves staff, parents, and friends. This process should take into account any necessary accommodations, including assistive technology.

Multiple and diverse community partnerships: Engagement of state systems and other organizations can be key to creating buy-in for the change process. Partners may include school districts, state agency offices such as vocational rehabilitation, faithbased and/or civic organizations, and transportation resources.

\subsection{Community life engagement}

Feedback from experts was synthesized into four main guideposts, which establish a set of quality indicators to be used as a roadmap as states and service providers focus their change efforts from traditional day programs towards meaningful CLE.

\subsubsection{Guidepost 1: Individualize supports for each person}

CLE supports should be tailored to the interests and needs of each unique person. In order to be individualized, supports must:

- Start with an understanding of personal preferences, goals, interests, and skills. Regardless of the process used to uncover interests and passions, the goal should always be the same: to identify and pursue activities that appeal to the individual and/or support their longer-term goals.

- Emphasize person-centered planning and discovery. Providers must take the time to get to know the individual through some form of person-centered planning or discovery, whether formal or informal. This includes allowing for an investigative period to uncover the most accurate and detailed information.

- Consider creative staffing, intentional grouping, and generating supplemental funding. One often-noted challenge is providing individualized supports when existing funding is based on group staffing ratios. To do this, service providers use strategies including creative and purposeful grouping of individuals; careful attention to scheduling, logistics, and staff communication; redefining staff roles to include community facilitation and to encourage natural supports; and accessing, braiding, or blending funding resources.

\subsubsection{Guidepost 2: Promote community membership and contribution}

In order to promote community membership and contribution, supports must:

- Start with inclusive settings and activities. Experts agreed that high-quality implementation means supporting people in inclusive environments alongside other adults without IDD. Accessing inclusive opportunities often involves service providers partnering with other local, non-disability-specific organizations to identify community resources and to generate new community-based options.

- Ensure staff presence does not limit connections with other community members. Staff presence should not interfere with developing relationships with community members. Experts described the need to retool staff training so that it is aligned with new expectations and new settings.

- Place value on not just presence, but membership in the community. This includes being known by people in one's community, forming relationships, and making a contribution to the community through work or volunteer activities.

- Consider individual preferences. A focus on community connections should not be pursued unilaterally for all people. Some individuals may prefer a less connected life, or already have a lot of connections through work, and that should be an option as long as it is an informed choice.

\subsubsection{Guidepost 3: Use human and social capital to decrease dependence on paid supports}

Individuals should be actively engaged in the community with minimal supports commensurate with their needs. In order to achieve this, CLE supports must:

- Use social capital to create natural supports. As individuals make more connections in their communities, the social capital they are building can be used as natural supports. Tapping into these 
natural supports enables fading of formal, paid supports.

- Teach skills to build human capital. Human capital refers to the specific skills an individual brings to his or her job and/or community. CLE activities can build individuals' human capital by teaching community access skills such as use of public transportation or fostering the development of soft skills (such as workplace etiquette and general professionalism) that can be used for employment. This initial investment in skillbuilding enables more fading of supports over time.

\subsubsection{Guidepost 4: Ensure that supports are outcome-oriented and regularly monitored}

In order to achieve outcomes such as life satisfaction, community membership and contribution, and decreased dependence on paid supports, CLE supports must be oriented toward, and monitored in relation to, those outcomes. The experts provided the following examples of how to do so:

- Emphasize goals rather than processes. When asked what constitutes quality CLE, experts focused on outcomes such as satisfaction, individualization, and connectedness to community, rather than on process measures such as times and locations of activities.

- Hold CLE supports to clear expectations and guidance. While being goal-oriented is the ideal, there is currently a lack of clarity around the goals of CLE in state and federal policy. There is a need for clearer guidance at the federal and state levels, as well as for service providers to specify their own values and expectations.

- Expect CLE to lead to or complement employment. The individual goals upon which each person's CLE supports are based should include age-appropriate roles in the community, with an emphasis on employment. CLE can supplement employment to create more of a full life, filling in any gaps in time, engagement, or interests.

\subsection{Starfire's outcomes}

Starfire's performance goals include (a) increasing the number of community relationships in persons with IDD's social network; (b) supporting people with IDD in attaining a regular valued social role in their community; and (c) actively engaging families in the building a stronger social network for their family member with IDD. Outcomes to date include:

- $77 \%$ of people with IDD do not have a valued social role in the community upon starting Starfire's program. After 150 hours of one-onone supports ( $\sim 1$ year) with Starfire, $89 \%$ attain a valued social role in the community such as a volunteer position, internship, or job by the end of that time.

- The average person with IDD has a network of 2 connections (unpaid, non-family, people without disabilities) at entry into the program. $92 \%$ of people with DD supported see an increase in their social network through new community relationships after 6 months.

- $86 \%$ of families of people with IDD supported are reported to be active in building their family member with IDD's social networks.

- $32 \%$ of people with IDD report a high quality of life at the start of the program. $64 \%$ of people with IDD report a high quality of life after 150 hours ( $\sim 1$ year) with Starfire.

- $92 \%$ of the jobs attained by people with IDD come from social connections built through Starfire's support.

\section{Discussion}

\subsection{Common themes and next steps across the research projects}

Consideration of the findings emerging from both research initiatives sheds light on several common themes fundamental to any organizational transformation effort, as well as to the vision of next-generation supports for people with IDD. Furthermore, our two separate research initiatives show employment and CLE are not mutually exclusive, but rather they are inextricably linked. The following themes are central to both transformation efforts.

One central theme is keeping the focus on each unique individual, their skills, passions, and preferences. For CLE, the first guidepost is to "individualize supports to each person" by learning about the individual and tailoring his or her supports and activities. Similarly, one of the ten elements essential in successful organizational change toward integrated employment is "an active, person-centered job placement process" that finds jobs for individuals, one person at a time. 
Another common theme is adopting a holistic perspective, rather than seeing employment and day supports as separate services. One of the ten elements for organizational change is "a holistic approach" that includes both employment and wrap-around supports. The corollary to this is the emphasis on CLE as leading to or complementing employment, which is part of CLE Guidepost 4.

A third theme is a maintaining a clear and consistent vision and goals. In fact, the Delphi panel ranked the establishment of "clear and consistent goals" and "an agency culture that supports values" as the two biggest priorities in organizational change. Likewise, Guidepost 4 indicates a need for both clear expectations and guidance in state and federal policy and a values-based orientation to all supports delivered at the service provider level.

Finally, both pieces of research indicate a need for organizations to develop new business models that support these values and approaches. Organizational transformation involves "reallocated and restructured resources" that move away from funding bricks and mortar to funding the development of creative, reengineered job descriptions for staff taking on the roles related to community based supports. Guidepost 1 includes creative approaches to scheduling, staffing, and communication to support flexible community-based CLE supports. Such creativity in new funding models shifts emphasis away from programs and structures to provide individualized options with the same financial resources.

These commonalities illustrate a need for service providers to focus their organizational change processes holistically across both employment and CLE supports. Both of these projects involve creation of toolkits for service providers and states. A next step for the ICI will be combining the lessons learned across both projects to create tools and technical assistance models for service provider capacity building toward converting to a new vision of community-based supports.

\subsection{Starfire's reflections}

Starfire's support program for people with IDD aims to "spring" people with IDD into community life so they are known by others for their gifts, not their disability. By connecting people with IDD to friends in the community, they see that their long-term quality of life and well-being improves. They also see people with IDD receiving more jobs and valued role opportunities through relationships they make in the community. Starfire's conviction is that all people no matter their challenges - can and should have a place in the community where they belong. Starfire is confidently heading toward a future for people with IDD that is tailored to fit their interests, skills, and passions, and leads toward a meaningful life filled with community relationships.

\section{Acknowledgments}

This manuscript was supported by the Administration on Intellectual and Developmental Disabilities, Administration for Community Living, U.S. Department of Health and Human Services (cooperative agreement \#90DN0295) and by the National Institute on Disability, Independent Living, and Rehabilitation Research, US Department of Health and Human Services (Field Initiated grant \#90IF0075 and Rehabilitation Research and Training Center grant \# 90RT5028-01-00). For more information on these projects, visit www.ThinkWork.org. Our thanks to the people who have contributed to these research projects, particularly Allison Cohen Hall, John Butterworth, Suzzanne Freeze, and Miwa Tanabe of the ICI; Hannah Curren from the UMass Boston School for Global Inclusion and Social Development; and Rie Kennedy-Lizotte and Adam Sass from the National Association of State Directors of DD Services. The opinions contained herein are those of the grantees and project participants and do not necessarily reflect those of the funders.

\section{Conflict of interest}

The authors have no conflict of interest to report.

\section{References}

Amado, A. N. (2013). Friends: Connecting people with disabilities and community members. Minneapolis, MN: University of Minnesota, Institute on Community Integration, Research and Training Center on Community Living.

Butterworth, J., Gandolfo, C., Revell, W. G., \& Inge, K. J. (2007). Community rehabilitation programs and organizational change: A mentor guide to increase customized employment outcomes. Training and Technical Assistance for Providers. Retrieved from www.communityinclusion. org/article.php?article_id=228 
Center for Medicare and Medicaid Services (2014). Home and community-based services. Retrieved from http:// www.medicaid.gov/Medicaid-CHIP-Program-Information/ By-Topics/Long-Term-Services-and-Support/Home-andCommunity-Based-Services/Home-and-Community-BasedServices.html

The Council on Quality and Leadership (2014). Toolkit for States. Retrieved from http://www.c-q-1.org/app/webroot/ files/DOCUMENTS/Toolkit\%20For\%20States\%202016. pdf

Hoff, D. (2014). Collaboration to promote self-determination: Analysis of Title IV of WIOA statue and proposed regulations and recommendations for regulatory changes. Retrieved from http://www.apse.org/wp-content/uploads/2015/06/CPSDWIOA-Title-IV-analysis.pdf

Inge, K. J., Wehman, P., Revell, G., Erickson, D., Butterworth, J., \& Gilmore, D. S. (2009). Survey results from a national survey of community rehabilitation providers holding special wage certificates. Journal of Vocational Rehabilitation, 30(2), 67-85.
Lindqvist, P., \& Nordänger, U. K. (2007). (Mis-?) using the E-Delphi Method: An attempt to articulate the practical knowledge of teaching. Journal of Research Methods and Methodological Issues, 1(1), 1-13.

Linstone, H. A., \& Turoff, M. (Eds.) (1975). The Delphi method: Techniques and applications. Boston, MA: Addison-Wesley.

U.S. Department of Justice (2011). Statement of the Department of Justice on enforcement of the integration mandate of Title II of the Americans with Disabilities Act and Olmstead v. L.C. Retrieved from http://www.ada.gov/olmstead/ q\&a_olmstead.htm

United States vs. State of Rhode Island (2014). Retrieved from http://www.ada.gov/olmstead/documents/ri-olmsteadstatewide-agreement.pdf

The White House, Office of the Press Secretary (2011). On Anniversary of Olmstead, Obama Administration Recommits to Assist Americans with Disabilities [Press release]. Retrieved from https://www.whitehouse.gov/the-press-office/ 2011/06/22/anniversary-olmstead-obama-administrationrecommits-assist-americans-dis 